\title{
Recommendations for the Evaluation of Cross-System Care Coordination from the VA State-of-the-art Working Group on VA/Non-VA Care
}

\author{
Kristin M. Mattocks, $P h D, M P H^{1,2}$, Kristin Cunningham, $P M P^{3}$, A. Rani Elwy, $P h D^{4,5}$, \\ Erin P. Finley, $P h D, M P H^{6,7}$, Clinton Greenstone, $M D^{3,8}$, Michelle A. Mengeling, Ph.D, 9, 10 , \\ Steven D. Pizer, PhD 17,12, Megan E. Vanneman, PhD, MPH ${ }^{13,14}$, Michael Weiner, MD, MP' ${ }^{15,16,17}$, and \\ Lori A. Bastian, MD, MPH ${ }^{18,19}$
}

\begin{abstract}
'VA Central Western Massachusetts Healthcare System, Leeds, MA, USA; ${ }^{2}$ Department of Quantitative Health Sciences, University of Massachusetts Medical School, Worcester, MA, USA; ${ }^{3}$ VHA Office of Community Care, Washington, DC, USA; ${ }^{4}$ Center for Healthcare Organization and Implementation Research, VA Boston Healthcare System, Boston, MA, USA; 5 Department of Psychiatry and Human Behavior, Alpert Medical

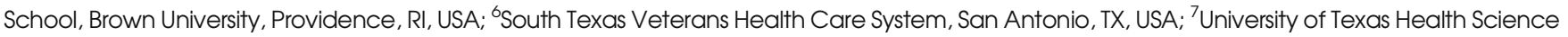

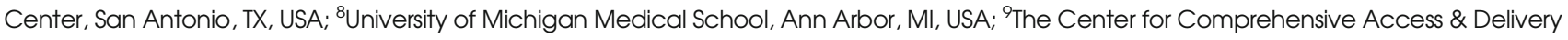
Research and Evaluation (CADRE) and VA Office of Rural Health (ORH), Veterans Rural Health Resource Center-lowa City (VRHRC-IC), lowa City VA Health Care System, lowa City, IA, USA; ${ }^{10}$ Department of Internal Medicine, University of lowa Carver College of Medicine, lowa City, IA, USA; ${ }^{11}$ VA Boston Healthcare System, Boston, MA, USA; ${ }^{12}$ Boston University School of Public Health, Boston, MA, USA; ${ }^{13}$ Informatics, DecisionEnhancement and Analytic Sciences Center, VA Salt Lake City Health Care System, Salt Lake City, UT, USA; ${ }^{4}$ Department of Internal Medicine/ Division of Epidemiology \& Department of Population Health Sciences/Division of Health System Innovation and Research, University of Utah School of Medicine, Salt Lake City, UT, USA; ${ }^{15}$ VA Health Services Research and Development Center for Health Information and Communication, Richard L. Roudebush VA Medical Center, Indianapolis, IN, USA; ${ }^{16}$ Regenstrief Institute, Inc., Indianapolis, IN, USA; ${ }^{17}$ Center for Health Services and Outcomes Research, Indiana University, Indianapolis, IN, USA; ${ }^{18}$ Department of Internal Medicine, Yale University School of Medicine, New Haven, CT, USA; ${ }^{19}$ Pain Research, Informatics, Multimorbidities, and Education (PRIME) Center, VA Connecticut Healthcare System, West Haven, CT, USA.
\end{abstract}

In response to widespread concerns regarding Veterans' access to VA care, Congress enacted the Veterans Access, Choice and Accountability Act of 2014, which required VA to establish the Veterans Choice Program (VCP). Since the inception of VCP, more than two million Veterans have received care from community providers, representing approximately $25 \%$ of Veterans enrolled in VA care. However, expanded access to non-VA care has created challenges in care coordination between VA and community health systems. In March 2018, the VA Health Services Research \& Development Service hosted a VA State of the Art conference (SOTA) focused on care coordination. The SOTA convened VA researchers, program directors, clinicians, and policy makers to identify knowledge gaps regarding care coordination within the VA and between VA and community systems of care. This article provides a summary and synthesis of relevant literature and provides recommendations generated from the SOTA about how to evaluate cross-system care coordination. Care coordination is typically evaluated using health outcomes including hospital readmissions and death; however, in cross-system evaluations of care coordination, measures such as access, cost, Veteran/patient and provider satisfaction (including with cross-system communication), comparable quality metrics, context (urban vs. rural), and patient complexity (medical and mental health conditions) need to be included to fully evaluate care coordination effectiveness. Future research should examine the role of multiple individuals coordinating VA and non-VA care, and how these coordinators work together to optimize coordination.

This manuscript has not been presented at any prior conference.

Published online May 16, 2019
KEY WORDS: Veterans; care coordination; health information interoperability; delivery of healthcare.

J Gen Intern Med 34(Suppl 1):S18-S23

DOI: $10.1007 /$ s11606-019-04972-1

(c) Society of General Internal Medicine (This is a U.S. government work and not under copyright protection in the U.S.; foreign copyright protection may apply) 2019

\section{INTRODUCTION}

Since its origins as a federal administration in 1930, the U.S. Department of Veterans Affairs (VA) has provided Veterans with comprehensive healthcare, including inpatient, outpatient, and long-term care. Much of this care is provided through VA's 170 medical centers and 1061 additional VA clinics. Beyond providing care directly through VA facilities, VA partners with federal, academically affiliated, and private providers, clinics, and hospitals to deliver care services for Veterans. Federal law allows the VA to pay for care at outside (non-VA) facilities via contract or individually authorized care arrangements, in which VA reimburses community providers for each service rendered to patients.

In response to widespread concerns regarding Veterans' access to VA care, however, Congress enacted the Veterans Access, Choice and Accountability Act of 2014, which required VA to establish the Veterans Choice Program (VCP). Veterans already enrolled in VA care that were waiting longer than 30 days for an appointment or lived more than 40 miles from the nearest VA medical facility became eligible to receive 
VA-paid non-VA care through a VA-approved provider. The VCP allowed VA to expand the availability of community care for eligible Veterans through enhanced relationships with community providers - including private practices and federally qualified health centers - and federal providers, including the Department of Defense and the Indian Health Service. In FY 2017, over 50\% of all Veterans Health Administration (VHA) community care appointments were delivered using the VCP. ${ }^{1}$ Although VCP and related non-VA care programs were intended to expand Veterans' access to care through community partnerships, these programs also posed challenges for coordination of care between VA and non-VA providers. Coordination problems related to making non-VA appointments, sharing electronic medical records between VA and non-VA systems, and timely receipt of results have all been well-documented. ${ }^{2,3}$ Thus, the chief goals of coordination are to improve health outcomes by ensuring that care from a multitude of providers is not provided in silos, and to help reduce healthcare costs by eliminating waste from the system.

In March 2018, the VA Health Services Research \& Development Service convened a State of the Art (SOTA) conference to review evidence about cross-system care coordination, identify related research and implementation gaps, and develop priorities for action. More than $50 \mathrm{VA}$ clinicians, researchers, and policy makers, as well as researchers outside the VA, participated in three workgroups to examine various aspects of care coordination, including care coordination within VA, measures and models of care coordination, and cross-system care coordination. This article summarizes findings from the workgroup focusing on coordination between VA and its nonVA partners. For the purposes of this SOTA workgroup, we focused solely on non-VA care paid for by the VA but provided by community providers, rather than care provided through other non-VA sources (e.g., private health insurance, Medicare, Medicaid).

\section{CURRENT EVIDENCE ON CROSS-SYSTEM CARE COORDINATION}

Prior to the SOTA, members of the non-VA care coordination group reviewed existing literature on cross-system care coordination in order to understand how other systems coordinated care for patients moving between two healthcare systems. ${ }^{4}$ In addition, members were provided with VHA definitions and models. The non-VA coordination group used the VA and non-VA literature to synthesize existing evidence and guide recommendations for improving non-VA care coordination. Previous studies have consistently found that VA performs as well or better than non-VA care in delivering high-quality care, although care quality varies across facilities and specialties. ${ }^{5,6}$

Studies characterizing Veterans' healthcare utilization indicate that as many as half of Veterans rely on both VA and nonVA sources of care, with older and more rural Veterans more likely to be dual users. ${ }^{7-9}$ Patients make complex decisions regarding healthcare seeking based on their access to care, perceptions of care, prior utilization, satisfaction, quality, and outcomes $^{10-12}$ and there is growing recognition of the need to assess patient experiences of cross-system care as a means of evaluating its coordination and quality. ${ }^{13}$ Both VA and nonVA providers have reported significant frustration with crosssystem care coordination, particularly related to barriers to information exchange and a lack of role clarity and care tracking. ${ }^{14-17}$ Health information technology (IT) has been shown to be an essential part of effective information exchange, but is itself highly complex and reflects only one component of achieving coordinated care. ${ }^{18,19}$

\section{MAJOR AREAS OF FOCUS FROM NON-VA CARE WORKGROUP}

To identify priority areas of focus, ideas were proposed and discussed by all workgroup members $(n=23)$. Through a consensus-building process, workgroup members chose to identify three areas of focus from the longer list of possible issues. The first was the need to identify which Veterans would benefit most from special efforts in coordinating care. With more than two million Veterans receiving non-VA care annually, some prioritization is necessary to coordinate care for Veterans most in need, requiring development of systems to identify which Veterans are most in need of care coordination. The second priority area focused on who should deliver care coordination services. Historically, primary care has been tasked with the responsibility to coordinate care for Veterans requiring non-VA care. However, organizational shifts within the VA have reorganized non-VA care coordination services into local offices of community care, raising questions regarding the most efficient and effective way to provide care coordination services. Finally, the non-VA care working group prioritized the need to identify or develop measurement systems that would accurately assess how effectively non-VA care is being coordinated with VA care for Veterans. An examination of each of these issues is detailed in the sections below.

\section{IDENTIFYING VETERANS WHO BENEFIT MOST FROM CARE COORDINATION}

VA is responsible for managing Veterans' care, whether that care is provided by VA providers or purchased from non-VA providers, but individual care coordination needs vary greatly. Veteran preferences, involvement of caregivers, health complexity, exigency, and access to healthcare affect care coordination needs. Veterans who have multiple chronic health conditions, who cannot fully participate in the coordination of their care, or who receive care across healthcare systems have the highest need for care coordination. ${ }^{20}$ Within VA, all Veterans are provided with a level of care coordination through their assigned Patient Aligned Care Teams (PACT), which are 
similar to patient-centered medical homes. However, higher levels of care coordination may be warranted due to greater healthcare need (e.g., multiple comorbidities, advanced or more severe conditions), limited personal resources, and frequent cross-system use. ${ }^{7,21-23}$ In addition to PACT, VA Office of Community Care has developed a triage tool to differentiate Veterans with low, moderate, or high needs for coordination to facilitate coordination and navigation within and outside the VA. This tool also helps in documenting the intensity ("dosage") of coordination delivered. In conjunction with VA's databases, researchers can use this tool to examine the relationship between care coordination and outcomes at the levels of Veteran, provider, and system. This system uses the Care Assessment Need (CAN) score as a base, and clinicians are able to upgrade or downgrade the care coordination needs based off other factors as well (e.g., social support).

\section{DELIVERY OF CARE COORDINATION SERVICES}

Deciding who coordinates Veterans' non-VA care is an important but potentially inconsistent aspect of VA care coordination efforts. Care coordination is often called a team sport, requiring the right infrastructure, resources, leadership, and culture to support synchronized efforts, communication, and collaboration among multidisciplinary teams of providers and specialists (NEJM Catalyst). Recent Medicare demonstration programs have examined the role care coordinators play in reducing re-hospitalization. ${ }^{4}$ By introducing the patient triage tool described above, VA is also paving the way for research to examine who is involved in care for specific patients, conditions, or cross-system care patterns (e.g., use of non-VA care primarily for women's or mental health), and to identify the composition of care coordination teams associated with best outcomes for patients. Specifically, care coordination is carried out at acute, chronic, post-discharge, and community levels, and may involve coordination efforts from hospital physicians, nurses, social workers, physical therapists, and primary care providers. For example, hospital physicians may communicate with primary care providers, physical therapists, and social workers to obtain advice on patient management, discharge plans, or follow-up care. Yet multidisciplinary coordination occurring in the absence of shared goals and relationships may impede the quality and timeliness of care and services. Thus, a framework for how to measure this care coordination is needed to ensure that all providers involved in care coordination efforts understand their shared roles and responsibilities and can meet the same goals of ensuring highquality care for veterans.

\section{CARE COORDINATION MEASUREMENT SYSTEMS}

Workgroup members identified six components necessary to include when measuring the efficacy of care coordination for non-VA care. Those elements include access, cost, satisfaction, quality of care, adequacy of local community provider networks, and timely sharing of medical records.

Access. Discussions of timely access to care often come with heightened emotions for both Veterans and the VA, since some VA medical centers, like many non-VA institutions around the country, have experienced struggles with timeliness of care. VA has developed and validated measures of access to VA care that are based on administrative scheduling data. ${ }^{24}$ Veterans can now find average wait times for appointments, specific to VHA facilities, clinics, and whether the Veteran will be a new or returning patient available online ("How Quickly Can My VA Facility See Me?"). ${ }^{25}$ However, this same information is not yet available for non-VA partners. Non-VA care introduces many elements that may remain outside VA's control, despite VA's authorization and sponsorship of such care. To identify and quickly address any backlogs that may arise, it is important to understand access at junctures across the scheduling process, including time from medical decision to referral; referral to appointment; appointment to availability of findings and recommendations; and results to follow-up care, whether VA or non-VA. To address this, VA is developing systems such as the HealthShare Referral Manager (HSRM). The HSRM is an online portal designed to collect information from multiple disparate systems to inform decision-making and track workflow. ${ }^{26}$ These new tools, unique to the VA, lend themselves to measuring important aspects of care coordination such as the impact of non-VA care on access.

Cost. Accurately measuring the cost of VA and non-VA care is equally challenging. The cost of non-VA care can be computed directly by adding the value of claims paid to community providers, but VA care is not administered through claims. Consequently, VA costs must be estimated by apportioning VA budgets to individual services. This is an accounting challenge, and the results are heavily influenced by decisions about what overhead costs are included, and the basis upon which those costs are apportioned. Researchers are currently developing and testing a method to compare costs between VA and non-VA care, toward the goal of supporting costconscious decision-making in planning and allocations for future care.

Satisfaction. One important consideration when evaluating VA and non-VA care coordination is Veteran satisfaction. Patient-centered care respects and responds to individual patients' preferences, needs, and values, while ensuring that the patient's values guide medical decisions. VA administers surveys modeled on Consumer Assessment of Healthcare Providers and Systems, through the Survey of Healthcare Experiences of Patients program, covering patient experience in VA general primary care, specialty care, and inpatient care, as well as for community care. In contrast to patient satisfaction, we are not aware of systematically collected data on 
provider satisfaction with care coordination. Provider satisfaction is of particular relevance in evaluating cross-system care coordination, because the timeintensiveness and complexity of such coordination can be a significant source of strain on providers, potentially increasing the likelihood of burnout and turnover among healthcare professionals, or reducing their willingness to invest their time in coordinating care.

Quality of Care. As Veterans increasingly seek care in places both inside and outside the VA, the risk of adverse events due to fragmentation of care may increase. We must study the quality of care, to know what specific aspects of VA's approaches to coordinating care may create special risks for Veterans. VA assesses 25 quality measures through their Strategic Analytics for Improvement and Learning Value Model, some of which focus on elements related to internal care coordination. ${ }^{27}$ The measures are a collection of metrics from various non-VA sources. While quality within VA is well-tracked, monitoring the quality of non-VA care is difficult, though the Office of Community Care is currently examining mechanisms to systematically evaluate non-VA care. For evaluating quality outcomes of care coordination, we highlight the following domains from the National Quality Forum's Preferred Practices and Performance Measures for Measuring and Reporting Care Coordination: A Consensus Report: healthcare home, proactive plan of care and follow-up, communication, information systems, and transitions or "handoff. ${ }^{28}$

Adequacy of Networks. When VCP was implemented, VA contracted with third-party administrators to improve access to non-VA provider networks. Early research of VCP implementation found that there were communication difficulties among stakeholders (e.g., VHA, third-party administrators, community providers, and patients), slow or incomplete exchange of medical records, network provider shortages, and concurrent barriers to community provider participation. ${ }^{3,14,17,29,30}$ The number of providers, providers' distribution across specialties (i.e., health professional shortage areas), locations of existing providers (e.g., clusters in urban vs. rural areas), and provider preferences (e.g., capacity to accept new patients, reimbursement limits) are not necessarily aligned with optimal care and coordination and so need further evaluation. Careful assessment of where current provider networks are inadequate, and for what specialties, will be required to support targeted network development toward the goal of increasing long-term capacity for delivery of community care.

Timely Sharing of Medical Records. Another key to coordinating care across public and private networks is timely sharing of medical records. VA and non-VA providers each need clinical information from the other, and VA administrators need to know if services referred to community networks were provided thoroughly and on time. VA has invested in health information exchange technology to support standardized methods to share records with community providers as well as the Department of Defense. Unfortunately, use of VA health information exchange has been low. ${ }^{10} \mathrm{Al}$ though some research has been done, ${ }^{31-36}$ additional research that measures aspects of care coordination via health information exchange would be helpful to policymakers and administrators who must allocate resources and balance privacy concerns against care coordination needs.

For additional discussion of care coordination measures and models, not limited to cross-system care coordination, see the conference's Measures and Models workgroup's manuscript elsewhere in this Supplement.

\section{FUTURE RESEARCH NEEDED}

Much research is needed to understand the impact of the VA's approach to coordinating care for its Veterans, and how to improve coordination of care further. For example, more studies are needed to evaluate the quality of care received across systems in the current era, although evidence suggests that dual-system care is more likely to be associated with care fragmentation, resulting in duplication or gaps in services and worse health outcome; ${ }^{37,38}$ these findings underscore the critical importance of care coordination to the success of VA's community care initiatives. Furthermore, we will need to examine and understand how medical centers have sought to establish infrastructure to facilitate coordination, what they established, decision-making (why, under what circumstances and indications) behind the cross-institutional communication and coordination, and where it happens (which places work together, how institutional relationships are forged and fostered, special challenges and solutions in urban or rural areas). Timeliness (when) is another critical element. We should examine whether adding non-VA care to the VA care of Veterans alters access and quality outcomes, whether positively or negatively, or whether outcomes are unchanged. In cases where differential outcomes become apparent, we will need to examine whether the difference may be attributed to the coordination process or events themselves, the specific medical conditions being addressed (e.g., mental illness), or the delivery of care. A related and important need is knowing when care should be provided outside the VA, perhaps due to availability or effectiveness of certain non-VA services. "Equitable" refers to "providing care that does not vary in quality because of personal characteristics such as gender, ethnicity, geographic location, and socioeconomic status." As we conduct the research described above, we will need to account for, and examine, these factors to gain an understanding of their effects on equity, ensuring VA's ability to support equitable care for all Veterans. 
Corresponding Author: Kristin M. Mattocks, PhD, MPH; VA Central Western Massachusetts Healthcare System, Leeds, MA, USA (e-mail: Kristin.Mattocks@va.gov).

Funding Information The work reported here was supported by the Department of Veterans Affairs, Veterans Health Administration, Office of Research and Development, and Health Services Research and Development \# SDR 17-156, CIN 13-407, and CIN 13-416. No contributors are listed for this manuscript.

\section{Compliance with Ethical Standards:}

Conflict of Interest: The authors declare that they do not have a conflict of interest.

Disclaimers: The views expressed in this article are those of the authors and do not necessarily represent the position or policy of the Department of Veterans Affairs or the United States government.

\section{REFERENCES}

1. Mattocks K, Yehia B. Evaluating the Veterans Choice Program: Lessons for developing a high performing integrated network. Med Care 2017; 55:1-3.

2. Mattocks KM, Yano EM, Brown AN, Casares JD, Bastian L. Examining women Veteran's experiences, perceptions and challenges with the Veterans Choice Program (VCP). Med Care 2018; 56:557560.

3. Mattocks KM, Mengeling $\mathbf{M}$, Sadler A, Baldor $\mathbf{R}$, Bastian $\mathbf{L}$. The Veterans Choice Act: A qualitative examination of rapid policy implementation in the Department of Veterans Affairs. Med Care 2017; 55:S71-S5.

4. Brown RS, Peikes D, Peterson G, Schore J, Razafindrakoto CM. Six features of Medicare coordinated care demonstration programs that cut hospital admissions of high-risk patients. Health Aff 2012;31(6):11561166.

5. O'Hanlon C, Huang C, Sloss E, et al. Comparing VA and Non-VA quality of care: A systematic review. J Gen Intern Med 2017;32(1):105-121.

6. Health R. Balancing Demand and Supply for Veterans' Health Care: A Summary of Three RAND Assessments Conducted Under the Veterans Choice Act. RAND Health 3 2016;6:12. https://doi.org/10.7249/ RR1165.4

7. Charlton ME, Mengeling MA, Schlichting JA, et al. Veteran use of health care systems in rural states: Comparing VA and Non-VA health care use among privately insured Veterans under age 65. J Rural Health 2016;32(4):407-417.

8. Hynes DM, Koelling $\mathbf{K}$, Stroupe $\mathbf{K}$, et al. Veterans' access to and use of Medicare and Veterans Affairs health care. Med Care 2007;45:214-223.

9. Finley EP, Mader M, Bollinger MJ, et al. Characteristics associated with utilization of VA and Non-VA care among Iraq and Afghanistan Veterans with Post-Traumatic Stress Disorder. Mil Med 2017;182(11):e1892e1903.

10. Byrne CM, Mercincavage LM, Bouhaddou O, et al. The Department of Veterans Affairs' (VA) implementation of the Virtual Lifetime Electronic Record (VLER): findings and lessons learned from health information exchange at 12 sites." Int J Med Inform 2014;83(8): 53747.

11. Fortney JC, Burgess JF, Bosworth HB, Booth BM, Kaboli PJ. A reconceptualization of access for 21 st century healthcare. J Gen Intern Med. 2011;26(Supplement 2):639-647.

12. Mengeling MA, Sadler AG, Torner J, Booth BM. Evolving comprehensive VA women's health care: patient characteristics, needs, and preferences. Womens Health Issues 2011;21(4):S120-S129.

13. Guinn M, Robinson C, Forman J, Krein SL, Rosland AM. Survey instruments to assess patient experiences with access and coordination across health care settings: Available and needed measures. Med Care 2017;55:S84-S91.

14. Zuchowski JL, Chrystal JG, Hamilton AB, et al. Coordinating care across health care systems for Veterans with gynecologic malignancies: a qualitative analysis. Med Care 2017;55:S53-S60.
15. Pope CA, Davis BH, Wine L, et al. Perceptions of U.S. Veterans Affairs and community healthcare providers regarding cross-system care for heart failure. Chronic Illness 2017;0(0):1-14 https://doi.org/10.1177/ 1742395317729887

16. Gaglioti A, Cozad A, Wittrock S, et al. Non-VA Primary Care providers' perspectives on comanagement for rural Veterans. Mil Med 2014; 179(11): 1236-1243.

17. Finley EP, Noèl PH, Mader M, et al. Community clinicians and the Veterans Choice Program for PTSD care: understanding provider interest during early implementation. Med Care 2017;55:S61-S70.

18. Wu FM, Shortell SM, Rundall TG, Bloom JR. The role of health information technology in advancing care management and coordination in accountable care organizations. Health Care Manag Rev 2017;42(4):282-291.

19. Hsiao CJ, King J, Hing $\mathbf{E}$, Simon AE. The role of health information technology in care coordination in the United States. Med Care 2015;53(2): 184-190.

20. McDonald KM, Sundaram V, Bravata DM, et al. Closing the quality gap: a critical analysis of quality improvement strategies (Vol. 7: Care Coordination). US Agency for Healthcare Research and Quality, 2017, http://www.ahrq.gov/downloads/pub/evidence/pdf/caregap/caregap. pdf. Accessed July 2018.

21. Pugh MJ, Finley EP, Wang CP, et al. A retrospective cohort study of comorbidity trajectories associated with traumatic brain injury in veterans of the Iraq and Afghanistan wars. Brain Inj 2016;30(12):148190.

22. Wilson NJ, Kizer KW. The VA health care system: an unrecognized national safety net. Health Aff 1997;16(4):200-4.

23. Agha Z, Lofgren RP, VanRuiswyk JV, Layde PM. Are patients at Veterans Affairs medical centers sicker?: A comparative analysis of health status and medical resource use. Arch Intern Med 2000; 160(21):3252-7.

24. Prentice JC, Davies ML, Pizer SD. Which outpatient wait-time measures are related to patient satisfaction? Am J Med Qual 2014; 29(3):227235.

25. “How Quickly Can My VA Facility See Me?" US Department of Veterans Affairs, Accessed 17 Dec 2018, https://www.accesstopwt.va.gov/Healthcare/Timeliness.

26. "VA's HealthShare Referral Manager is Good News for Community Providers" US Department of Veterans Affairs, Accessed 17 Dec 2018, https://www.va.gov/COMMUNITYCARE/docs/providers/HSRM_Information_Sheet.pdf

27. "Strategic Analytics for Improvement and Learning (SAIL) Value Model Measure Definitions" US Department of Veterans Affairs, Accessed 10 Dec 2018, https://www.va.gov/QUALITYOFCARE/measure-up/SAIL_definitions.asp

28. National Quality Forum (NQF), Preferred practices and performance measures for measuring and reporting care coordination: a consensus report, Washington, DC: NQF; 2010. Accessed 5 Dec 2018.

29. Gellad WF, Cunningham FE, Good CB, et al. Pharmacy use in the first year of the Veterans Choice Program: a mixed-methods evaluation. Med Care 2017;55:S26-S32.

30. Ball SL, Stevenson LD, Ladebue AC, McCreight MS, Lawrence EC, Oestreich T, et al. Adaptation of Lean Six Sigma Methodologies for the Evaluation of Veterans Choice Program at 3 Urban Veterans Affairs Medical Centers. Med Care 2017;55:S76-S83.

31. Byrne C, Hunolt E, Bouhaddou $\mathbf{O}$, et al. Performance evaluation framework for the Virtual Lifetime Electronic Record (VLER) health information exchange pilot program. AMIA Symp 2013; 162.

32. Dixon BE, Haggstrom DA, Weiner M. Implications for informatics given expanding access to care for Veterans and other populations. J Am Med Inform Assoc 2015 ; 22(4):917-920.

33. French DD, Dixon BE, Perkins SM, et al. Short term medical costs of a VA health information exchange: a CHEERS-compliant article. Medicine 2016; 95(2):1-5.

34. Dixon BE, Ofner S, Perkins SM, et al. Which Veterans enroll in a VA health information exchange program?, J Am Med Inform Assoc2017; 24(1):96-105.

35. Nguyen KA, Haggstrom DA, Ofner S, et al. Medication use among Veterans across health care systems. Applied Clinical Informatics 2017; 8(1):235-249.

36. Weiner M, Haggstrom DA. Health information exchange in the VA. VA HSR\&D Forum, May 2013, page 5. Available at <http://www.hsrd. research.va.gov/publications/forum/Apr13/apr13-4.cfm> (29 April 2013). 
37. Liu CF, Chapko M, Bryson CL, et al. Use of outpatient care in Veterans Health Administration and Medicare among Veterans receiving primary care in community-nased and hospital outpatient clinics: Outpatient care in VA and Medicare. Health Serv Res. 2010;45(5p 1): 1268-1286.

38. Van Walraven $\mathbf{C}$, Oake $\mathbf{N}$, Jennings A, Forster AJ. The association between continuity of care and outcomes: a systematic and critical review: Association between continuity of care and outcomes. J Eval Clin Pract 2010;16(5):947-956.
Publisher's Note Springer Nature remains neutral with regard to jurisdictional claims in published maps and institutional affiliations. 